\title{
ADAPTIVE ASSESSMENT IN WEB-BASED LEARNING *
}

\author{
Dunwei Wen ${ }^{1}$, Sabine Graf ${ }^{2}$, Chung Hsien Lan ${ }^{3}$, Terry Anderson ${ }^{1}$, Kinshuk ${ }^{1}$, Ken Dickson ${ }^{1}$ \\ ${ }^{1}$ Athabasca University, 1 University Drive, Athabasca, AB T9S 3A3, Canada \\ ${ }^{2}$ Women's Postgraduate College for Internet Technologies, Vienna University of Technology, Austria \\ ${ }^{3}$ Nanya Institute of Technology, Jhongli, Taiwan
}

\begin{abstract}
Web-based assessment is used in different contexts with the aim to support students and help to make learning easier and more effective for them. Typically, the individual characteristics and needs of students are used to personalize and customize existing approaches to assessment. In this paper, we show the potential of adaptive web-based assessment in different learning applications. We introduce adaptive systems in the area of readiness self-assessment, performance self-assessment, and peer assessment. We show how they incorporate individual differences and create adaptive applications.
\end{abstract}

\section{INTRODUCTION}

Web-based assessment is widely used to support students in learning and help them to achieve their learning goals. For example, readiness self-assessments are often used in open universities to help students determine if they have the necessary skills and attitudes for successful completion of a course or program. Another application of web-based assessment includes assessment of the learning process itself. Self-assessment tests are commonly used in technology enhanced environments, especially in learning management systems. Students can use such self-assessment tests to check their acquired knowledge and get feedback about their learning progress. Another pedagogical strategy in web-based learning includes peer assessment where students are asked to assess peers' assignments. Such web based tests can be used in distance education systems where it is impractical to assume that students can gather simultaneously in a common space. These applications can be enhanced when the assessment is customized to the individual student resulting in higher motivation and time efficiencies for both students and assessors [10].

In all these cases, adaptivity plays a supportive role. As Brusilovsky [1] argued, learners have different needs and

\footnotetext{
* This research has been funded partly by the Austrian Federal Ministry for Education, Science, and Culture, and the European Social Fund (ESF) under grant 31.963/46-VII/9/2002.
}

these differences should also be considered in web-based education. In web-based assessment, this can be achieved in different ways. In the following sections, we present three novel approaches from different domains which show how adaptive web-based assessment can support students.

\section{READINESS SELF-ASSESSMENT}

Online readiness self-assessments are widely used in universities that provide open and distant education service, to help prospective students to identify the learning style and the requirements of studying as a distant learning student. Most current readiness self-assessment tools are online questionnaires. The new version of AM I READY for Athabasca University is an adaptive online self-assessment tool (http://my2.athabascau.ca/amiready/). It is based on several knowledge models about the counseling process. It involves better understanding of a user and creates an effective self-assessment process with a smaller question set than the previous self-assessment system.

\subsection{Related work}

Adaptive questionnaires and self-assessments have been the subject of considerable research, much of which focuses on development and maintenance of an effective student model. An adaptive web-based questionnaire for course survey is developed and evaluated by Chou et al. [2]. They use the answers to some key questions, which are called Adaptive Questions, to determine the next series of questions and to skip unrelated questions. Nokelainen et al. [3] designed an adaptive questionnaire based on Bayesian Modeling, which is suitable for surveys or assessments whose categories and outcomes are not well known. More recent works on knowledge modeling have focused on a visual formalism and an evaluation tool, XDM, context-sensitive dialogue modeling, to model user-adapted interface based on extended Petri nets [4]. Adaptive Testing [5], in which testing is adapted interactively to match the ability level of the examinee by means of a statistical method called "Item Response Theory".

\subsection{Knowledge modeling}


We use User Model to represent the knowledge about users, and model the counselor by Counseling \& Process Model (knowledge about the counseling process), and Assessment Model (knowledge about the assessment). Figure 1 shows the models and the overall system architecture.

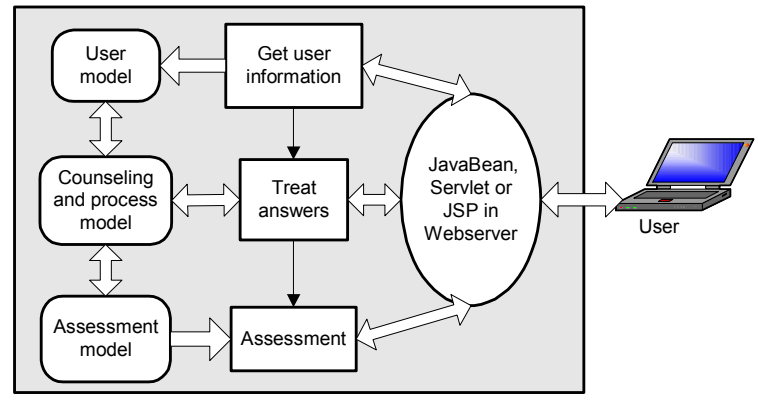

Figure 1. Knowledge Models and Architecture

User Model: Although there is much information about a user, we only need some of it that mostly affects the readiness self-assessment of the user. We divide it into two types, namely static user model, which is acquired at the beginning of the self-assessment, and dynamic user model, which responds to the user's choices and is always changing during the assessment.

Counseling \& Process Model: We have captured several kinds of knowledge for Counseling \& Process Model in AM I READY, which include Questions, Relations, and Instant Information. Questions are the knowledge sets about all questions of a system, its fields and sub-fields, and predefined answer types. All questions are divided into eight groups (or fields) in AM I READY. Relations (or Rules) include the relations between questions, which can be used to control the conselling process as well as the questions sets asked by the system. The relations here are always one-tomany relations such as question to question(s), question to sub-field(s). The latter can finally become question to all questions belonging to the sub-field(s). We introduced four types of relations, which are Enable, Disable, Plus, and Contradictory. The first three relations are in the following form:

(Enable (Answer( $i$ ) of question(j), (question list), (sub-field list)))

Enable means that an answer to a question will enable some specified questions or all questions belonging to specified sub-fields if they have been disabled before. Disable relation can disable some questions that are no longer needed to ask according to the user's current answer. Plus means to enable some questions and add the priority of those questions as well, which can cause those questions to be asked sooner than other questions. Contradictory means that an answer, say, Answer( $i$ ) of question( $j)$, is contradictory to some other answers, say, list of answers of some questions in (2):

(Contradict (Answer( $i$ ) of question(j), ( list of answers of some questions) ) )
Instant Information is the additional multimedia information related to answers of certain questions. Users can see the instant information immediately after they click an answer button, which can give them immediate response and provide them with related resources and guidance to follow the self-assessment process. This turns the assessment process into an explicit learning process.

Assessment Model: We do not use a single score to assess the readiness of a user. Instead, we provide some assessment information plus remedial suggestions to help potential students understand their current readiness and steps to take to enhance their probability of success. In this model, we distinguished two kinds of assessment information, Question Related and Question Group Related, to support the final assessment. The latter has more than one conditions coming from different fields and questions:

\section{(Assessment $(i) \leftarrow$} (answer $(i)$ of question $(j), \ldots \ldots)$ )

The final assessment information is displayed in the end. Users can browse, download, email, and print the assessment information in AM I READY system.

By the above models, AM I READY can choose questions by filtering, sorting and skipping, and ask the chosen questions more relevant to its users. It behaves in a way that the users are "understood" to some extent and suitable or natural "dialog" between users and the system is then performed. Figure 2 shows the interface of the system.

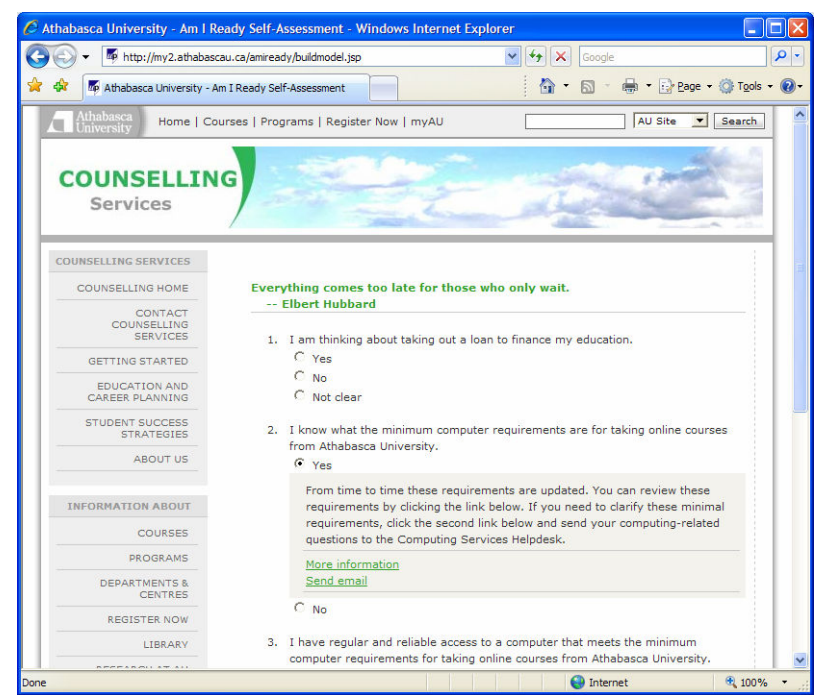

Figure 2. The interface of self-assessment

\section{PERFORMANCE SELF-ASSESSMENT}

In this section, we describe a novel approach to provide adaptivity based on learning styles in learning management systems. Adaptivity includes presenting different kinds of learning objects. Self-assessment in the form of theoretical questions and exercises plays a central role in determining 
the choice and order of these objects. Self-assessment can be used by students to test their already acquired knowledge, whereas exercises provide students with opportunities to practice. Both kinds of tests help students in learning by giving them feedback and showing students their progress in the course.

\subsection{Related work}

Adaptivity in web-based systems is motivated by educational and psychological theories. For example, Felder [6] pointed out that learners with a strong preference for a specific learning style may have difficulties in learning if the teaching style does not match with their learning style. On the other hand, incorporating learning styles makes learning easier and leads to better achievement. Bajraktarevic, et al. [7], for example, confirmed this by a study showing that students attending an online course that matches with their preferred learning style (either sequential or global) achieved significantly better results than those who got delivered a course that did not match their learning style.

\subsection{Adaptive features for self-assessment}

Each learner has a different way of learning and therefore the preference for and the use of self-assessment is different. In our approach, we consider learning styles according to the Felder-Silverman learning style model [6], where each learner has a preference for the active/reflective, sensing/ intuitive, visual/verbal, and sequential/global dimension.

The theoretical tests can be adapted in terms of their position in the course. They can be presented at the beginning of each chapter, at the end of each chapter and/or at the end of the course. Intuitive learners like challenges, whereas sensing learners prefer to solve problems by standard approaches they have learned before. Therefore, sensing learners get presented tests after the presentation of the learning material, whereas for intuitive learners, such tests are presented at the beginning of each chapter. Since active learners like to try things out and work actively, we present tests at the beginning and at the end of each chapter. In contrast, for reflective learners, we present tests only at the end of the course. Because the learning progress of sequential learners is linear, they prefer to be tested in shorter intervals than global learners who need more time to get the big picture of a topic. For the latter, tests at the end of the course are more suitable while for the former, tests are provided at the end of each chapter.

Regarding exercises, we adapted the number and the position in the course. For sensing learners, the number of exercises should be high since they tend to like practical problem solving, whereas for intuitive learners the number of exercises decreases because they do not like repetition. As for the theoretical tests, exercises are presented at the beginning of a chapter for intuitive learners and at the end of a chapter for sensing learners. For active learners, it does not matter where exercises are presented, but they prefer to solve many of them. In contrast, reflective learners focus more on the learning material and therefore we decrease the number of exercises and present them at the end of each chapter. For sequential and global learners, we do not adapt the number of exercises but we avoid presenting exercises at the beginning of the chapter for global learners since for them it is important to get first the big picture of the topic before solving tasks.

\subsection{Self-assessment for improving adaptivity}

Providing adaptivity requires knowing the needs of learners. Self-assessment tests act as a good source to get information about the learning styles of students in order to create and then update the student model and therefore improve adaptivity. Several patterns exist, for example, the time students spent on tests gives indications about the sensing/intuitive preference, since sensing learners are known to be more careful and therefore take more time for revising and controlling their answers. Another example is the number of performed exercises that gives an indication about the preference for active/reflective learning. Multimedia object and questions about the content presented by such multimedia objects can be used to identify a visual or verbal preference. A sequential or global preference can be indicated by the performance of questions about details and overview.

While the adaptation features are currently evaluated by a course with about 400 students, further investigations about the concept of updating the student model by data from the behavior of students are necessary.

\section{PEER ASSESSMENT}

Peer assessment is an interactive assessment method that enhances student interpretation and reflection, enabling instructors and the students themselves to improve their understanding of student performance. Students are capable of learning how to critique peer work and accept peer criticism, thereby developing their critical thinking skills and self-reinforcement through peer assessment.

Peer assessment is one form of group assessment, which can include student involvement not only in the final judgment made of student work, but also in the prior setting of criteria and the selection of evidence of achievement. However, the issue of fairness has to be concerned in group assessment. For example, the awarding of grades may not accurately reflect the individual student's achievement due to subjective judgment, bias or insufficient assessment ability. The proposed methodology can aggregate students' marks to reduce personal bias and consider individual learning styles of students who give marks into the assessment process in order to enhance the accuracy of the 
assessment. Thus, the aggregated assessment considering students' learning styles provides students a better feedback.

\subsection{Related work}

Peer assessment is a widely adopted technique that can be applied to improve learning processes [8]. Web-based environments typically enable students to develop their individual learning portfolios and conveniently assess those of their peers. A web-based peer assessment system successfully assists students in developing their understanding. Students' achievement increased significantly as a result of the peer assessment process and the number of students willing to take part in learning activities also significantly increased [9]. Lin et al. [10] used aptitude treatment interaction design to examine how executive thinking styles affect web-based peer assessment and indicated that students with high executive thinking styles significantly improved the effectiveness of peer assessment. In our study, learning styles are considered to enhance the accuracy of peer assessment.

\subsection{Adaptive peer assessment}

Peer assessment requires cognitive activities such as reviewing, summarizing, clarifying, providing feedback, diagnosing errors, and identifying missing knowledge or deviations. However, each student has an individual learning style and thus has different abilities to assess peers' work. The adaptive peer assessment process can be divided into three separate stages. In stage 1, peer reviewers fill out a questionnaire in order to detect their learning styles (active/reflective, sensing/intuitive, visual/verbal, and sequential/global) [6]. In stage 2, an assessment model provides assessment criteria and includes all relations between these criteria and learning styles. In our experiment, there are four assessment criteria, which are Creativity, Completeness, Execution, and Security, to assess peer assignments in a Database System course. Peer reviewers provide their marks for different assessment criteria. After the reviewers generate preliminary scores and feedback, an aggregate model summarizes all marks and sends the result to the original author who then revises the original assignment based on peer feedback in stage 3 . The aggregate function is in this form:

$$
\text { Assessment feedback }=w_{1} x_{1}+w_{2} x_{2}+\ldots w_{\mathrm{i}} x_{\mathrm{i}} \text {, }
$$

where $x_{1}, x_{2, \ldots}, x_{\mathrm{i}}$ are assessment criteria and $w_{1}, w_{2}, \ldots, w_{\mathrm{i}}$ represent the importance or weight in aggregating all marks according to different dimensions in learning styles. For example, active students tend to be experimentalists and thus are more familiar with execution and security. Therefore, the importance of assessment provided by active students can be seen as higher than by reflective students. Sensing students like solving problems by standard methods and are patient with detail. Therefore, their marks in completeness are more important. Intuitive students like innovation, which can be very helpful for assessment in creativity.

By this process, students typically develop a more serious attitude toward their coursework. According to the different learning styles, we develop a flexible aggregate model to summarize marks in order to improve the quality of peer assessment.

\section{CONCLUSION}

This paper introduced different applications in the area of adaptive web-based assessment by reviewing systems developed for readiness self-assessment, performance selfassessment, and peer assessment. These systems show different ways of providing adaptivity and also incorporate different needs and characteristics of students. By improving systems with adaptivity, the assessment can be done more effectively and students can benefit more from the assessment. As discussed, these benefits are relevant to many educational applications, for example, assigning grades in peer assessment, showing students their learning progress or helping them with the decision whether distance learning is the right choice for them.

\section{REFERENCES}

[1] Brusilovsky, P. "Adaptive Hypermedia", User Modeling and User Adapted Interaction, 2001, 11(1/2), 87-110.

[2] Chou, C., Chang, Y-F., Jiang, Y-Y. "The Development of an Online Adaptive Questionnaire for Health Education in Taiwan", Computers \& Education, 2000, 35(3), 209-222.

[3] Nokelainen, P., Niemivirta, M., etc. "Bayesian Modeling Approach to Implement an Adaptive Questionnaire", World Conference on Educational Multimedia, Hypermedia and Telecommunications, 2001, 1, 1412-1413.

[4] Rosis, F., Pizzutilo, S., Carolis, B. D. "Formal Description and Evaluation of User-Adapted Interfaces", Int. J. Human-Computer Studies, 1998, 49(2), 95-120.

[5] Jettmar, E., Nass, C. "Adaptive Testing: Effects on User Performance", Conference on Human Factors in Computing System, Minneapolis, Minnesota, USA, 2002, 129-134.

[6] Felder, R.M., Silverman, L.K. "Learning and teaching styles in engineering education”, Engineering Education, 1988, 78(7), 674681 .

[7] Bajraktarevic, N., Hall, W., \& Fullick, P. "Incorporating learning styles in hypermedia environment: Empirical evaluation." Workshop on Adaptive Hypermedia and Adaptive Web-Based Systems, Nottingham, UK, 2003, 41-52.

[8] Freeman, N. "Peer assessment by groups of group work", Assessment and Evaluation in Higher Education, 1995, 20, 289300 .

[9] Liu, E. Z. F., Lin, S. S. J., Chiu, C. H., Yuan, S. M. "Student participation in computer sciences courses via the Network Peer Assessment System", Advanced Research in Computers and Communications in Education, 1999, 2, 744-747.

[10] Lin, S. S. J., Liu, E. Z. F., Yuan, S. M., "Web-based peer assessment: feedback for students with various thinking-styles", Journal of Computer Assisted Learning, 2001, 17 (4), 420-432. 